\title{
Smart Sensor Network based Industrial Parameters Monitoring in IOT Environment using Virtual Instrumentation Server
}

\author{
https://doi.org/10.3991/ijoe.v13i11.7630 \\ Nagarjuna Telagam ${ }^{(\varpi)}$,Nehru Kandasamy \\ Institute of Aeronautical Engineering, Hyderabad, India \\ nagarjuna473@gmail.com \\ Menakadevi Nanjundan \\ Hindustan College of Engineering and Technology, Coimbatore, India \\ Anuradha Thotakuri \\ MLR Institute of Technology, India.
}

\begin{abstract}
A remote monitoring and control are one of the most important criteria for maximizing the production in any industry. With the development of modern industry the requirement for industrial monitoring system is getting higher. This project explains the real time scenario of monitoring temperature and humidity in industries. National Instruments my RIO is used and results are observed on LabVIEW front panel and VI Server. The server VI program and client VI program is developed in block diagram for the two sensor data. This proposed system develops a sensor interface device essential for sensor data acquisition of industrial Wireless Sensor Networks (WSN) in Internet of Things (IOT) environment. By detecting the values of sensors like temperature, humidity present in the industrial area. The results are displayed on the web page. The data can be accessed with admin name and password. After logging into the web page the index of files is displayed. After restarting the my RIO kit and initiate the deploying process the file the excel sheet will appear on the VI Server. This VI server is tested for its working, using a data acquisition web application using a standard web browser. The critical situation can be avoided and preventive measures are successfully implemented.
\end{abstract}

Keywords-Sensors, my RIO, IOT, LabVIEW, VI Server, Excel Sheet.

\section{Introduction}

Nowadays web server based monitoring systems are widely used in many industries, and they set up a PC-based server which consumes a large power. This project explains data acquisition system that is controlled by an ARM processor and a web server application [1]. This paper proposes S3C2440 32 bit ARM Processor with Linux porting within built DM9000 Ethernet controller. Finally the application is 
developed and ported into an ARM9 processor using embedded ' $\mathrm{C}$ ' language. Web pages are written by Hypertext markup language (HTML) [2]. Remote Monitoring, Control and intelligent are one of the most important criteria for maximizing production and process plant availability. This data acquisition and control system (DACS) measure the remote signals and controls the remote devices through reliable protocols and communication network as a web server and also the addition of a GSM mobile communication will help to provide ubiquitous access to the system when the web server is not available to client [3]. This paper presents an implementation of a platform-independent embedded web server and its integration into a network of wireless sensor nodes. It allows authorized Internet users to establish two-way communication with the sensor network. The server uses limited available hardware resources to implement an interface to the WSN node and to serve dynamic HTML pages to the remote user [4]. In this paper, the embedded web server, which takes Samsung Corporation's ARM9-S3C2440AL processor as core, its operating system is Linux the system hardware architecture is presented. Then the process of the Linux operating system being transported on ARM is introduced [5]. The main objective of the proposed architecture is to be interfaced various sensors to measure the sensor analog data and displayed in LabVIEW on the monitor using the graphical user interface (GUI) [6]. Sensor data is integration in embedded based system explained through data programming model [7]. Figure 1 explains the architecture of IOT. This proposed system was designed mainly in three layers. The first layer is a data acquisition layer which the sensor data is deployed in more and second layer is the internet layer which can be used in cloud computing and third and foremost layer deals with application layer in which the user can monitor the data with client and server base model.

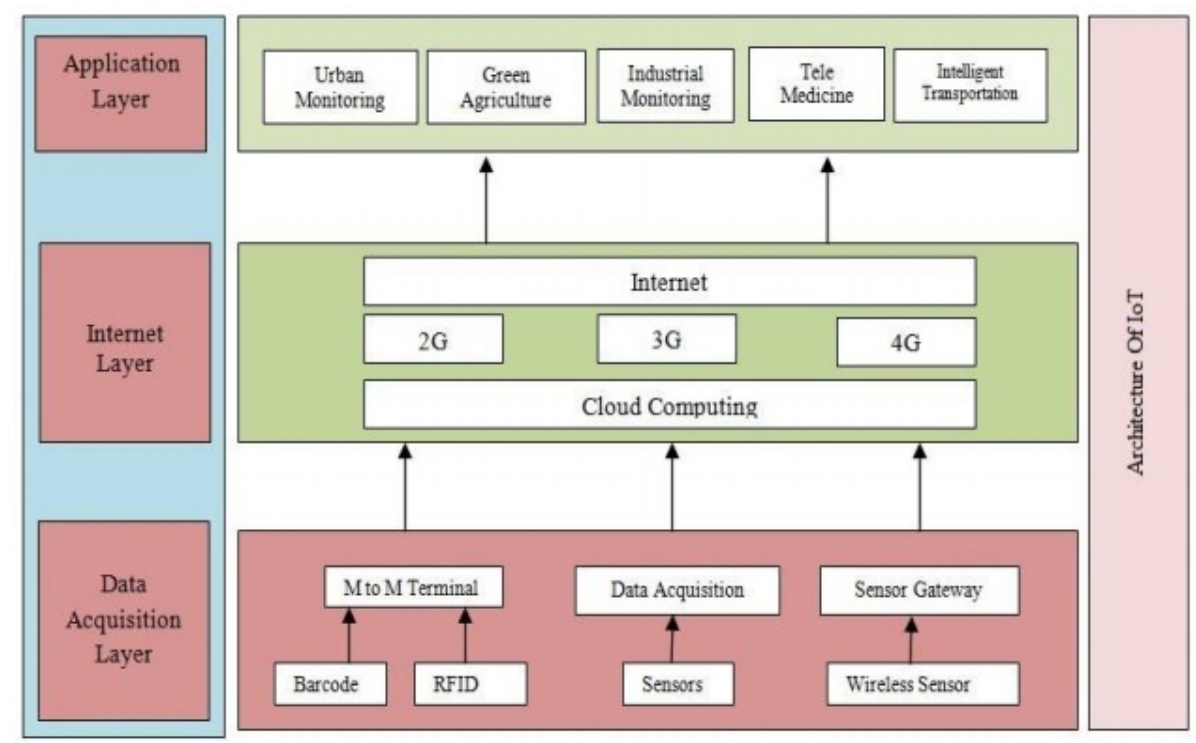

Fig. 1. Architecture of Internet of Things 


\section{Implementation}

Fig 2 shows the block diagram of the proposed system in which the humidity sensor named DHT 11 and temperature sensor named LM35 is integrated to my RIO through a bread board connection. The LED will glow and not glow depends on the threshold value given in the server program. The results can be viewed using the VI server.

Fig 3 shows the connection of bread board to my RIO. As the figure LED is glowing because the room temperature exceeds the threshold value. My RIO has the capability to integrate 10 input analog signals and provide 6 analog output lines. It also has integrated WIFI which the user can control it through the mobile phone.

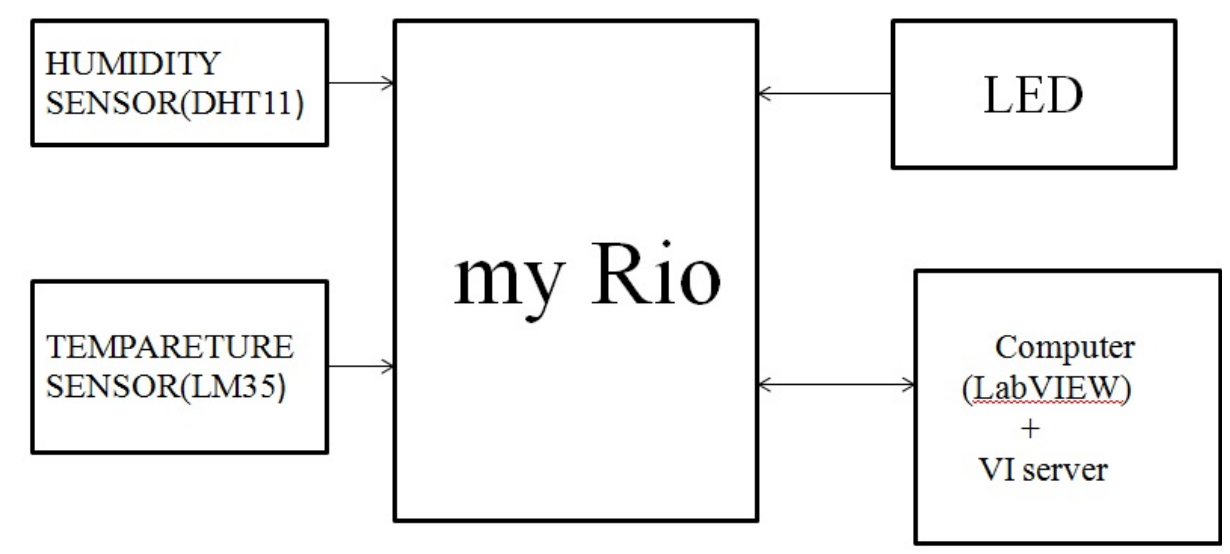

Fig. 2. Block diagram of proposed system using my RIO
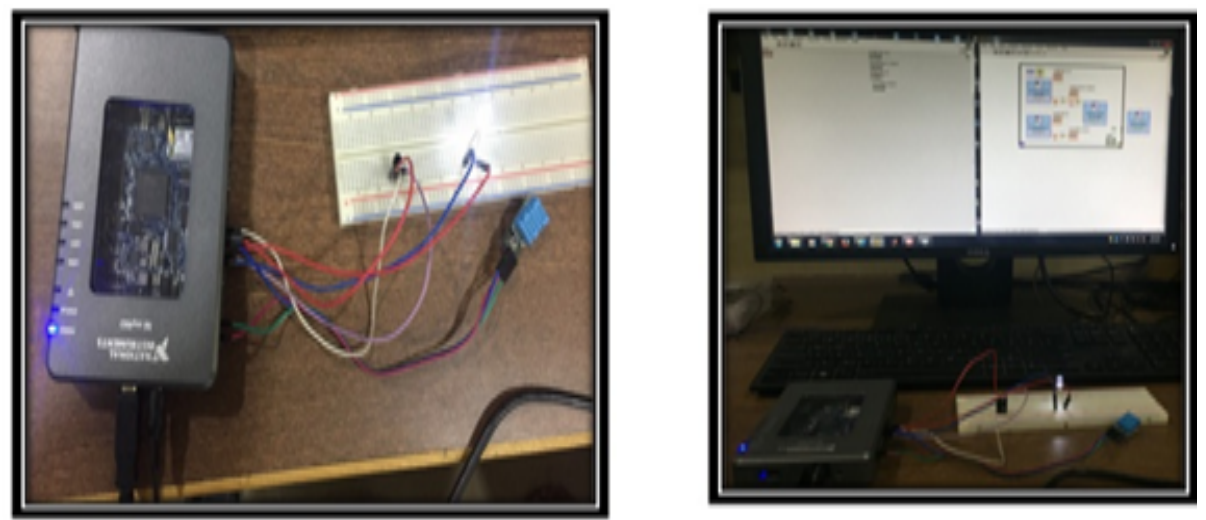

Fig. 3. Integration of my RIO to PC with bread board connection of sensors 


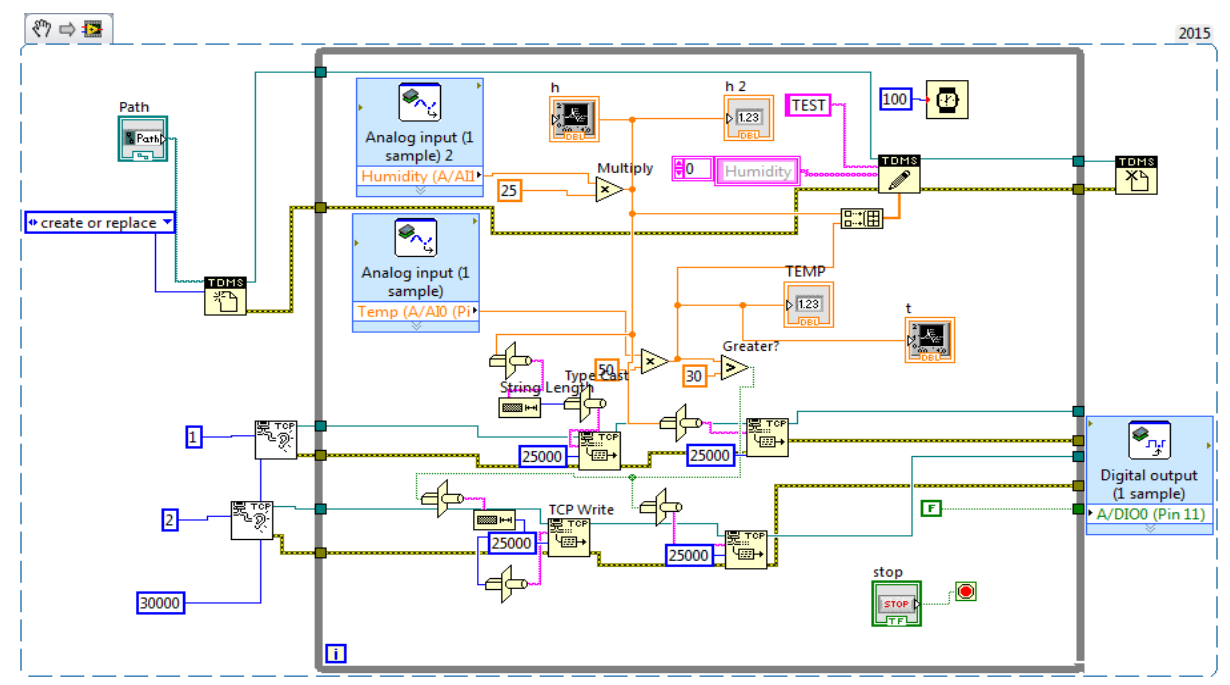

Fig. 4. Server VI Snippet using TCP protocol

This the server program in the block diagram panel, which TCP listen is used with timed out. The analog input 1 st and 2 nd samples are temperature and humidity sensor values are connected to the output. With the help of type cast the numerical value is converted into string data type and given to TCP Write. The yellow color data lines specify the error. This entire VI runs in a while loop with a time delay of $100 \mathrm{~ms}$.

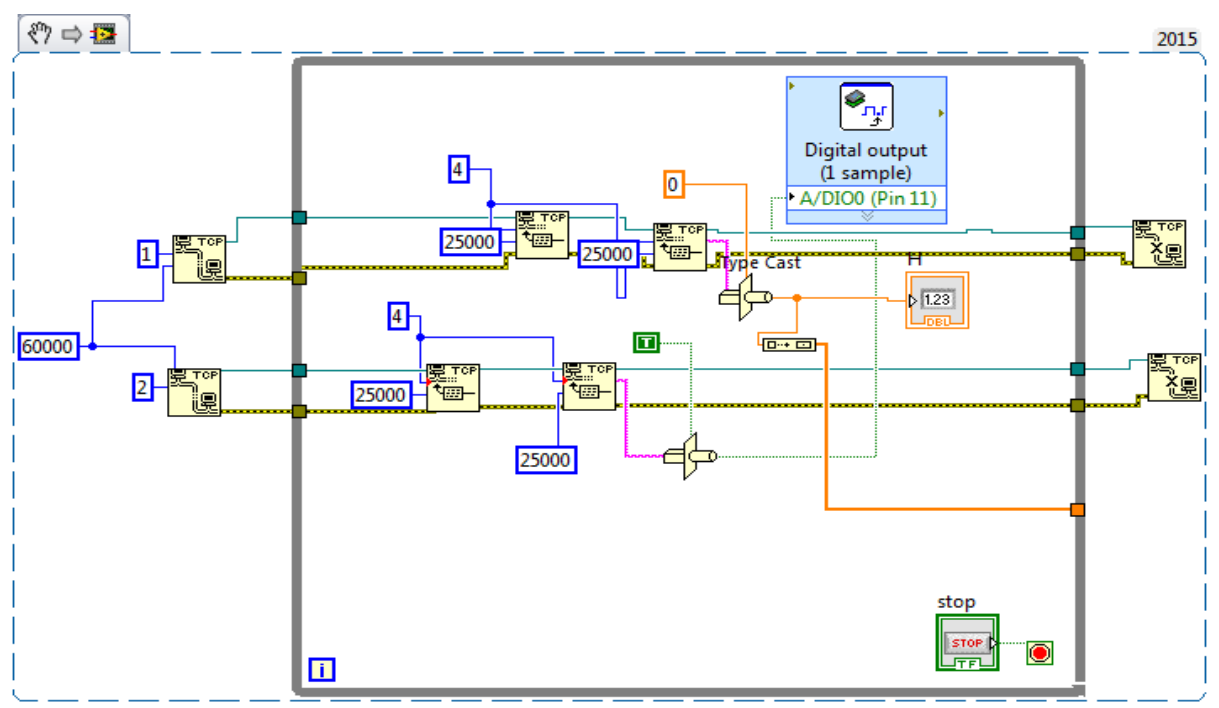

Fig. 5. Client VI Snippet using TCP Protocol

This VI program starts with TCP open connection with timed out. TCP read is having string data and is connected to type cast to convert into a numerical value of hu- 
midity sensor. We created a file path in the desktop with TDMS open, write and close to display the data in excel sheet. Similarly, another TCP read is used to display the temperature sensor data in the front panel.

\section{$3 \quad$ Results}

After designing the server and client VI's run them simultaneously at the same time, so that the output is observed on the front panels. Next, go the web browser and type 172.22.11.2/ files and search. The site says NI Auth is required. It will ask username and password. Just type admin in username and leave the password empty and go to login. It will display set of index files.

Figure 8 shows the data of humidity and temperature sensors. In the room temperature, the humidity sensor displays data around 62 (units are grams of water vapor per cubic meter volume of air) and temperature sensor displays around $27 \mathrm{de}-$ grees. TDMS file can help us to display the data also.

To the index of files go the lv user and natinst and you will find parent directory and read me file paths.txt. The data were not available at that time. Next again go to my RIO properties and check the TCP/IP connection is verified or not. Again restart my RIO and deploying process again. Visit the webpage again and click refresh button so that log.csv file is available in the web page. Then the user can have that file on their computer and double clicking on the file excel sheet will open which gives the values of humidity and temperature sensor values at different times.

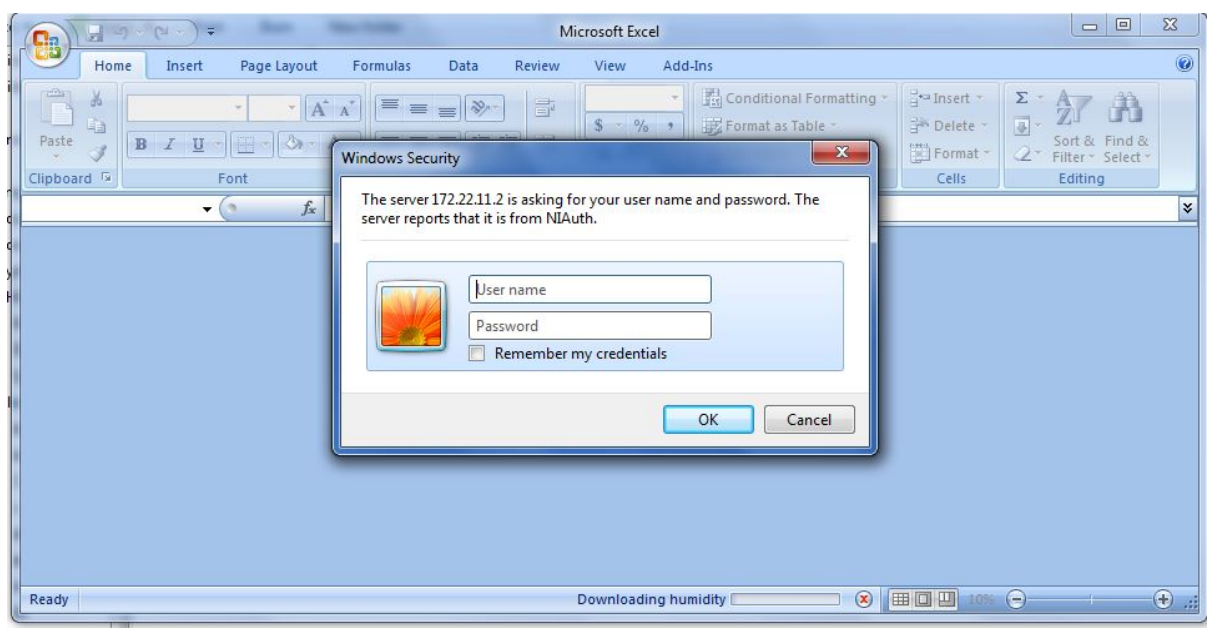

Fig. 6. Client Interaction in web browser with NI authorization 


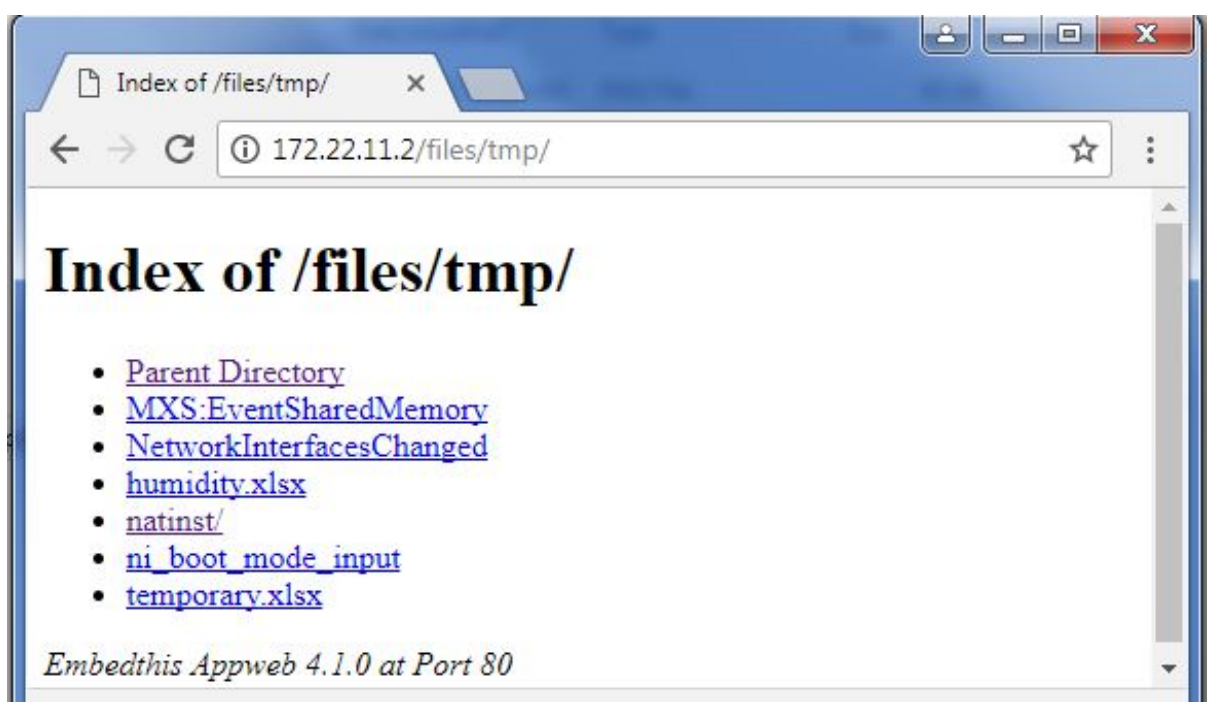

Fig. 7. Web page displays data after deploying my RIO with sensors data

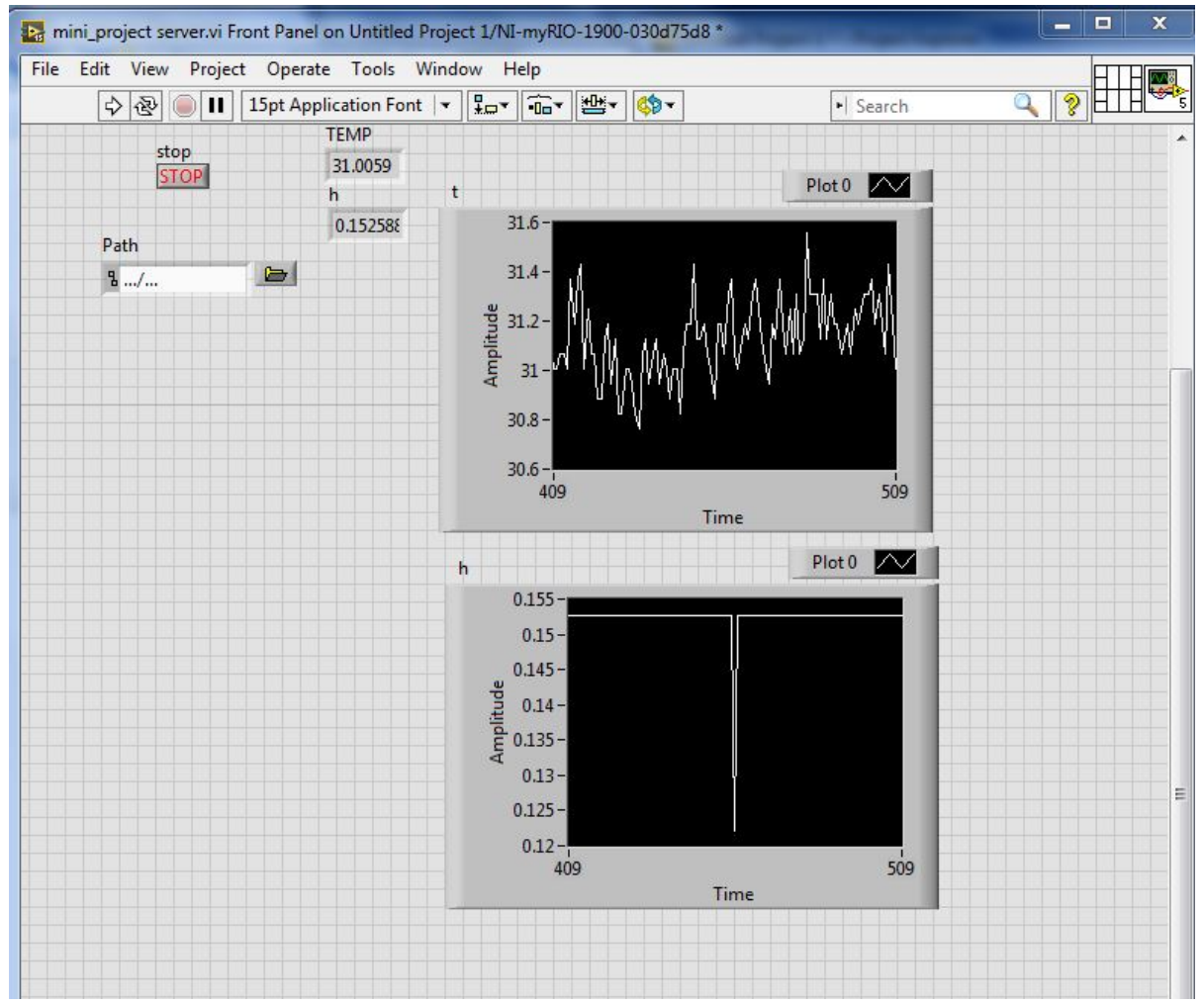

Fig. 8. Front panel showing the output graphs of humidity and temperature sensors. 


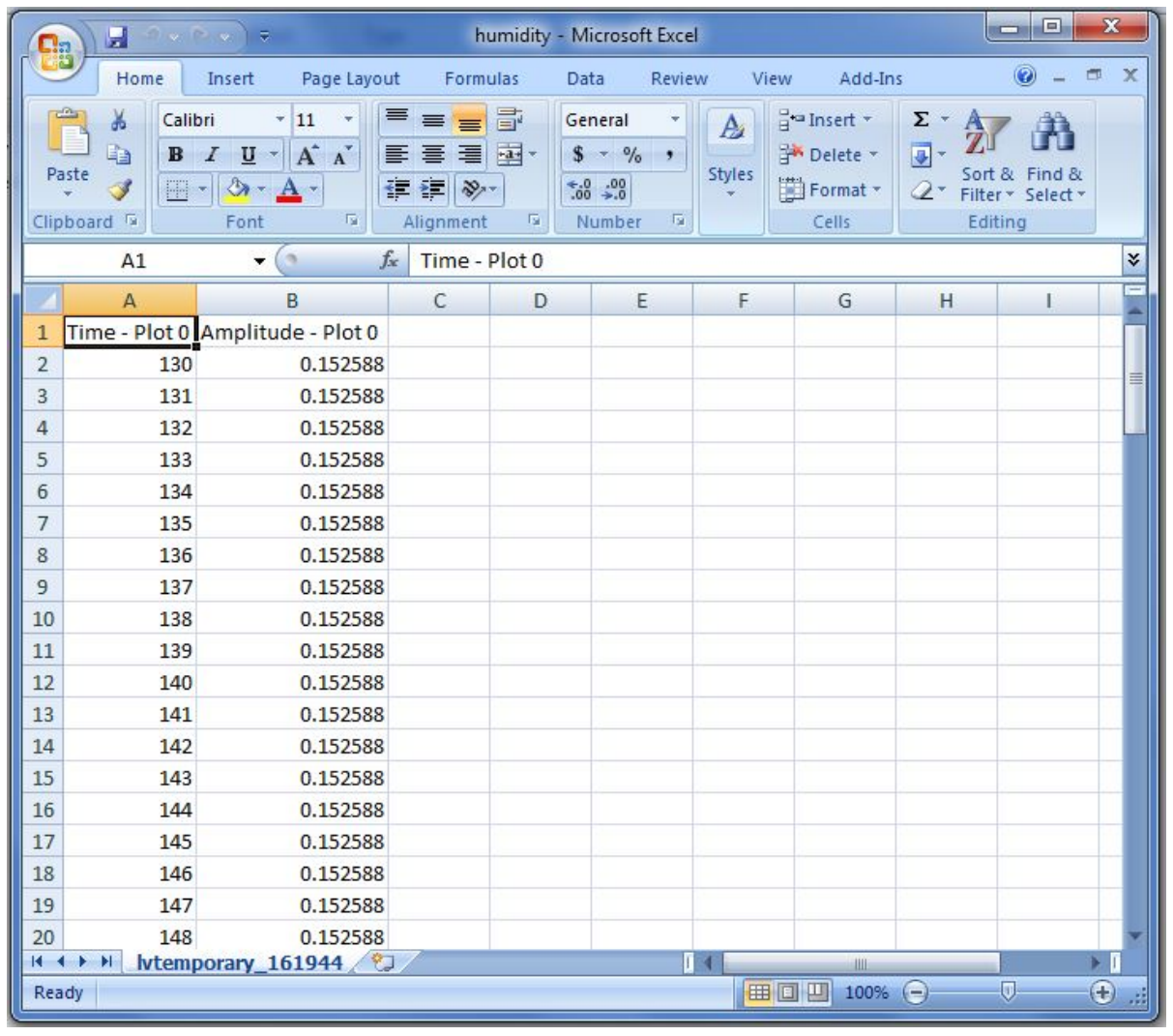

Fig. 9. Excel Sheet displays the sensors data with date and time

Table 1. Comparison of VI Server with Other Web Servers.

\begin{tabular}{|l|l|l|l|}
\hline \multicolumn{1}{|c|}{ Parameter } & \multicolumn{1}{|c|}{ Embedded web server } & \multicolumn{1}{c|}{$\begin{array}{c}\text { Traditional web } \\
\text { server }\end{array}$} & \multicolumn{1}{c|}{ VI Server } \\
\hline Size & Small & Large & Very small \\
\hline Cost & Effective $(\$ 55)$ & Costly (about \$600) & No Cost \\
\hline $\begin{array}{l}\text { Energy consump- } \\
\text { tion }\end{array}$ & $5 \mathrm{~W}$ & $>250 \mathrm{~W}$ & $0 \mathrm{~W}$ \\
\hline portability & Yes & No & Yes \\
\hline Heat sink & Not Required & Required & Not Required \\
\hline performance & Good(UsesARM-11Processor) & Good & $\begin{array}{l}\text { Very Good (uses Intel Core } \\
\text { i7-7700K) }\end{array}$ \\
\hline
\end{tabular}

\section{Conclusion}

With the rapid increase in the design and development in the field of industrial process controlling and monitoring. It is necessary to make a higher demand of the data accuracy. This my RIO system can adapt to the strict requirements of the data 
acquisition and control system such as the function, reliability, cost, size and remote access. The VI server mode is used to share the data with clients in online.

\section{$5 \quad$ References}

[1] P.Ferrari, A. Flammini, D. Marioli, E. Sisinni, A. Taroni.(2005) An Internet based interactive embedded data acquisition system for real time application, IEEE Trans. Instrument measurement, 54( 6).

[2] Manivannan M, Kumaresan N(2011), Design of on-line interactive data acquisition and control System for Embedded real time application, Proc. IEEE International Conference on Emerging Trends in Electrical and Computer Technology, pp. 551-556. https://doi.org/10.1109/ICETECT.2011.5760179

[3] Prof.S.S.Wagh, Pradeep V.Kalge(2013), Remote monitoring and control of industrial parameters Using Embedded web server and GSM, Indian Journal of applied research, vol. 3,pp 214.

[4] Dejan Raskovic, Venkatramana Revuri, David Giessel and Aleksandar Milenkovic(2009), Embedded web server for wireless sensor network, IEEE 41st Southeastern Symposium on System Theory, March 15-17, pp.19- 23.

[5] Mo Guan, Minghai Gu(2012), Design and Implementation of an Embedded Web server based on ARM, IEEE International Conference on Computer Science Education, pp 479482.

[6] Telagam Nagarjuna, Nehru Kandasamy, Nagendra Prasad, Menakadevi Nanjundan (2017). Smart Sensor Network Based High Quality Air Pollution Monitoring System Using Labview. International Journal of Online Engineering, 13(8), pp .79-87, https://doi.org/10.3991/ijoe.v13i08.7161.

[7] Telagam Nagarjuna, Menakadevi Nanjundan, Nehru Kandasamy, Soma Naidu(2017). Cruise Control of Phase Irrigation Motor Using Spark Fun Sensor. International Journal of Online Engineering, 13(8), pp .192-198. https://doi.org/10.3991/ijoe.v13i08.7318

\section{Authors}

Nagarjuna Telagam (corresponding author) is with the Electronics and Communication Engineering Department, Institute of Aeronautical Engineering, Hyderabad, India. Currently he is Ph.D Research Scholar in Sathyabama University, Chennai and is interested in the following topics: Wireless Communications, MIMO, OFDM, GFDM. Currently, He is working as Assistant Professor. He received his Bachelor degree from JNTU University/ Narayana Engineering College in 2011. He received his Master degree from Anna University/ Loyola Institute of Technology in 2013. He is Anna University rank holder (20) for M.E degree in2013.He has nearly 4 years of experience in teaching and research. He published 15 papers in scopus indexed Journals. He is certified LabVIEW Associate Developer. (nagarjuna473@gmail.com).

Nehru Kandasamy received his Bachelor Degree in Erode Sengunthar Engineering College, Anna University in 2005. And he obtained his Master Degree in R.M.K Engineering, Anna University in 2007. And he also obtained outstanding master student at that year. He is obtained his Ph.D in 2014 at Faculty of Information and Communication Engineering, Anna University, India. His main research interest is in 
the area of Low Power VLSI, Testing of VLSI Circuits, FPGA Design, CAD for VLSI, Signal processing. He has published papers on these topics in various international journals.(nnehruk@gmail.com).

Menakadevi Nanjundan is with the Electronics and Communication Engineering Department, Hindustan College of Engineering and Technology, Coimbatore, India. She is a Research Fellow and is interested in the following topics: Digital Image processing. She completed Master degree in Loyola institute of technology/Anna University Chennai in the year 2013. She is Anna University Rank holder (22) for her M.E degree. She finished her Bachelor degree B.E - electronics and communication engineering in muthayammal engineering college in 2010 She published 5 papers in Scopus Indexed journals. (menakadevin@gmail.com)

Anuradha Thotakuri is with the Electronics and Communication Engineering Department, MLR institute of Technology. (manthenaanuradha@gmail.com)

Article submitted 30 August 2017. Published as resubmitted by the authors 11 October 2017. 